\section{Prolonged Intake of Isoflavone- and Saponin- Containing Soybean Extract (Nijiru) Supplement Enhances Circulating $\gamma-$ Carboxylated Osteocalcin Concentrations in Healthy Individuals}

\author{
Masayoshi Yamaguchi, ${ }^{* a}$ Rie Ono, ${ }^{a, b}$ \\ and Zhong Jie $\mathrm{Ma}^{a}$
}

\begin{abstract}
${ }^{a}$ Laboratory of Endocrinology and Molecular Metabolism, Graduate School of Nutritional Sciences, University of Shizuoka, 52-1 Yada, Shizuoka 422-8526, Japan and ${ }^{b}$ Department of Research and Development, Marumiya K.K., 2211 Uchida, Kikusui-machi, Tamana-gun, Kumamoto 865-0104, Japan
\end{abstract}

(Received July 24, 2001; Accepted August 9, 2001)

The effect of nijiru, which is a by-product of the processing of soybeans to make the fermented soybeans called natto, on circulating blood chemistry levels related to calcium and bone metabolism in healthy individuals was investigated. Twelve volunteers (six men and six women) were received nijiru twice a day for 60 days at a dose of $1500 \mathrm{mg}$ ( 6 tablets) per day. The serum $\gamma$-carboxylated osteocalcin concentration was significantly increased by the intake of nijiru in both men and women to about 2 -fold that in the control group. The serum calcium concentration was significantly decreased by nijiru supplementation in women, and the serum inorganic phosphorus concentration was significantly reduced in both men and women. However, the intake of nijiru did not have a significant effect on serum glucose, nitrogen urea, albumin, free cholesterol, triglyceride, high-density lipoprotein cholesterol, and $\gamma$-glutamyltranspeptidase concentrations in men or women, indicating that liver and renal function is not affected by nijiru supplementation. The results of the present study suggest that the intake of isoflavone- and saponin-containing nijiru can stimulate the $\gamma$-carboxylation of osteocalsin, which plays an important role in bone formation and mineralization, in healthy individuals.

*To whom correspondence should be addressed: Laboratory of Endocrinology and Molecular Metabolism, Graduate School of Nutritional Sciences, University of Shizuoka, 52-1 Yada, Shizuoka 422-8526, Japan. Tel. \& Fax: +81-54-264-5580; Email:yamaguch@u-shizuoka-ken.ac.jp
Key words - genistein, saponin, $\gamma$-carboxylated osteocalcin, bone metabolism, osteoporosis

\section{INTRODUCTION}

It is known that bone mass decreases in both men and women with increasing age. The decrease in bone mass is due to increased bone resorption and to decreased bone formation. Osteoporosis with a decrease in bone mass is widely recognized as a major public health problem. The most dramatic expression of this disease is represented by fractures of the proximal femur, of which the incidence increases as the population ages. ${ }^{1,2)}$ A deficiency in both micronutrients and macronutrients appears to be strongly implicated in the pathogenesis and consequences of hip fracture in the osteoporotic elderly. ${ }^{3)}$ Nutritional factors may prevent bone loss with increasing age, although this has not been fully clarified.

Daidzein and genistein, natural isoflavonoid phytoestrogens found in Leguminosae, have an anabolic effect on bone metabolism in rats. ${ }^{4-6)}$ Isoflavones including daidzin, daidzein, genistin, and genistein are present in soybeans high concentrations. Daidzin and genistin are hydrolyzed to daidzein and genistein, respectively, by $\beta$-glucosidase in the gastrointestinal tract. Genistein and daidzein have been demonstrated to stimulate bone formation and to inhibit bone resorption, thereby increasing bone mass. ${ }^{7-13)}$

Nijiru is produced in the processing of the fermented soybean preparation natto, and contains significant quantities of isoflavone and saponin. The intake of isoflavone- and saponin-containing nijiru has been found to have an anabolic effect on bone components in rats, suggesting its role in the prevention of osteoporosis. ${ }^{14)}$ Soybean saponin has been demonstrated to have an anabolic effect on bone component in vitro and in vivo. ${ }^{15)}$ Moreover, the prolonged intake of natto supplemented with nijiru containing isoflavone and saponin has a preventive effect on ovariectomy-induced bone loss, suggesting that it may have a role in the prevention of osteoporosis. ${ }^{16)}$ Thus soybean-based foods may be useful for the prevention of osteoporosis with increasing age.

The present study was undertaken to determine the effect of supplemental nijiru intake on bone metabolic marker in healthy individuals over the long term. 


\section{MATERIALS AND METHODS}

Materials — Soybeans were boiled under 1.5 atmospheric pressure for $40 \mathrm{~min}$ at $160^{\circ} \mathrm{C}$, and the nijiru produced was then freeze-dried. The isoflavone content was measured after removal from the nijiru powder by extraction with $80 \%$ hot ethanol solution. This ethanol solution was filtered, and the filtrate was subjected to reverse-phase high-performance liquid chromato-graphy. The isoflavone concentration was expressed as micrograms per gram of nijiru powder. The calcium content in the nijiru powder was measured by atomic absorption spectrophotometry after digestion with $\mathrm{HNO}_{3}$. The composistion of saponin, daidzin, daidzein, genistin, genistein, and calcium in nijiru prepared from soybeans was $320,1800,12,1600,15$, and $820 \mu \mathrm{g} / \mathrm{g}$ dry powder, respectively. One tablet contained $250 \mathrm{mg}$ of nijiru dry powder.

Experimental Procedures — Twelve adults aged 17-58 years (six men and six women), who were judged to be healthy with no abnormal liver or kidney function as assayed by standard biochemical data, were enrolled as volunteers in this study. Informed consent was obtained from all. The intake of other foods with an abundance of vitamin K, which stimulates production of $\gamma$-carboxylated osteocalcin, was prohibited during the study. All volunteers were $1500 \mathrm{mg}$ (6 tablets) of nijiru powder twice a day for 60 days. Blood samples were collected at 10:00 on the day prior to the beginning of administration and 60 days thereafter. Serum $\gamma$ carboxylated osteocalcin, calcium, and inorganic phosphorus levels and other biochemical levels were measured using routine methods.

Analytical Procedures — Serum samples were obtained by centrifugation ( $2500 \mathrm{rpm}$ for $5 \mathrm{~min}$ ) between 20 and 40 min after blood collection, and then stored at $-20^{\circ} \mathrm{C}$ until assayed. The serum $\gamma$-carboxylated osteocalcin concentration was assayed using a Gla-type Osteocalcin (Gla-OC) EIA Kit (Takara Shuzou, Shiga, Japan). ${ }^{17)}$ Serum calcium and inorganic phosphorus concentrations were determined using KIT (Wako Junyaku, Osaka, Japan). Serum glucose, nitrogen urea, albumin, free-cholesterol, triglyceride, high-density lipoprotein (HDL) cholesterol, and $\gamma$-GTP were assayed using KIT.

Statistical Analysis — Differences in values before and after the intake of nijiru tablets were estimated using Student's $t$-test. $p$ values of less than 0.05 were considered statistically significant.
Table 1. Change in Serum Calcium and Inorganic Phosphorus Concentrations Following Intake of Isoflavone- and Saponin-Containing Soybean Extract in Healthy Individuals

\begin{tabular}{|c|c|c|}
\hline Serum level & $\begin{array}{c}\text { Control } \\
\text { (baseline) }\end{array}$ & At day 60 \\
\hline \multicolumn{3}{|c|}{ Calcium (mg/dl) } \\
\hline All & $9.00 \pm 0.09$ & $8.61 \pm 0.21$ \\
\hline Men & $9.18 \pm 0.12$ & $9.02 \pm 0.29$ \\
\hline Women & $8.81 \pm 0.08$ & $8.28 \pm 0.25^{*}$ \\
\hline \multicolumn{3}{|c|}{ Inorganic phosphorus $(\mathrm{mg} / \mathrm{dl})$} \\
\hline All & $3.36 \pm 0.12$ & $4.02 \pm 0.16^{* * * *}$ \\
\hline Men & $3.12 \pm 0.15$ & $3.89 \pm 0.27 * *$ \\
\hline Women & $3.60 \pm 0.15$ & $4.22 \pm 0.07 * *$ \\
\hline
\end{tabular}

Table 2. Serum Metabolic Findings Following Intake of Isoflavone- and Saponin-Containing Soybean Extract in Healthy Individuals

\begin{tabular}{lrr}
\hline \hline Serum level & $\begin{array}{c}\text { Control } \\
\text { (baseline) }\end{array}$ & At day 60 \\
\hline Glucose $(\mathrm{mg} / \mathrm{dl})$ & $100.3 \pm 4.00$ & $91.7 \pm 4.67$ \\
Nitrogen urea (mg/dl) & $14.1 \pm 0.96$ & $13.4 \pm 1.08$ \\
Albumin $(\mathrm{g} / \mathrm{dl})$ & $5.1 \pm 0.11$ & $5.1 \pm 0.08$ \\
Free cholesterol (mg/dl) & $49.0 \pm 4.61$ & $44.8 \pm 2.23$ \\
Triglyceride $(\mathrm{mg} / \mathrm{dl})$ & $56.9 \pm 5.68$ & $67.2 \pm 8.06$ \\
HDL cholesterol (mg/dl) & $55.3 \pm 3.36$ & $59.1 \pm 3.30$ \\
$\gamma$-GTP $(\mathrm{IU} / \mathrm{l})$ & $32.9 \pm 4.81$ & $29.7 \pm 5.06$ \\
\hline \multicolumn{2}{c}{ Each value is the mean \pm S.E.M. of 12 healthy volunteers. }
\end{tabular}

\section{RESULTS}

The changes in serum calcium and inorganic phosphorus concentrations in healthy individuals before and after administration of nijiru are shown in Table 1. The serum calcium concentration was significantly decreased with the intake of nijiru tablets in women, although not seen in men. The serum inorganic phosphorus concentration was significantly reduced in both men and women at 60 days compared with baseline.

The effect of nijiru tablet intake on serum metabolic findings is shown in Table 2. There was no significant alteration in glucose, nitrogen urea, albumin, free-cholesterol, triglyceride, HDL cholesterol, and $\gamma$-GTP levels in either men or women.

The changes in serum $\gamma$-carboxylated osteocalcin concentration after 60 days of nijiru intake are shown in Fig. 1. Serum $\gamma$-carboxylated osteocalcin increased significantly in individuals of both sexes. 


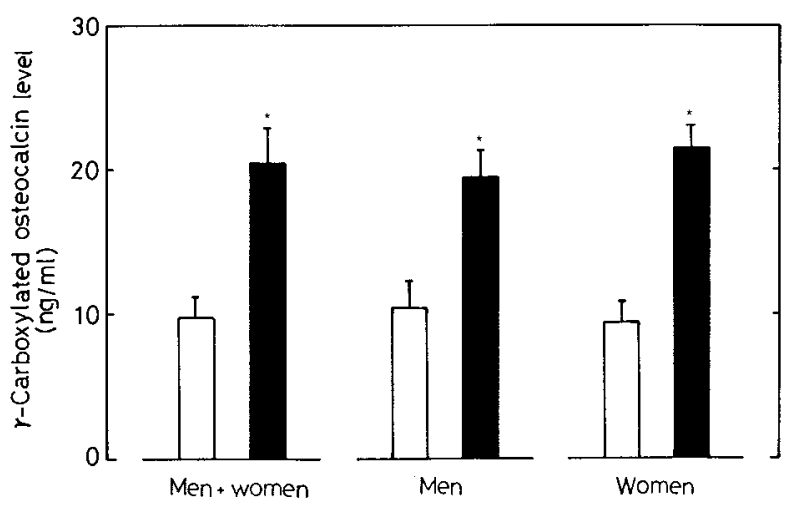

Fig. 1. Changes in Serum $\gamma$-Carboxylated Osteocalcin Concentrations Following Intake of Isoflavone- and Saponin-Containing Soybean Extract

Each value is the mean \pm S.E.M. of 12 (men and women) or 6 (men or women) individuals. ${ }^{*} p<0.01$ compared with the baseline value. White bars, control; black bars, after nijiru intake.

\section{DISCUSSION}

Nutritional factors may be significant in preventing bone loss with increasing age. Soybean isoflavone has been shown to have an anabolic effect on bone metabolism in rats, suggesting its role in prevention of osteoporosis. ${ }^{4-13)}$ Nijiru has been demonstrated to have a stimulatory effect on bone formation and mineralization in normal rats and an inhibitory effect on bone loss in ovariectomized rats. ${ }^{14-16)}$ We found that the intake of nijiru supplement causes a significant increase in circulating $\gamma$ carboxylated osteocalcin concentration in healthy individuals.

Osteocalcin is a bone matrix protein containing $\gamma$-carboxyglutamic acids, which is synthesized in osteoblasts of bone tissues. ${ }^{18,19)}$ Noncarboxylated osteocalcin cannot bind to hydroxyapatite in mineralized tissues. ${ }^{20)}$ Nijiru contains large quantities of isoflavones (genistin, genistein, daidzin, and daidzein). ${ }^{14)}$ These isoflavones have a stimulatory effect on protein synthesis in osteoblastic MC3T3E1 cells. ${ }^{7-9)}$ The intake of nijiru supplement caused a significant increase in the circulating $\gamma$-carboxylated osteocalcin concentration in healthy individuals. Presumably isoflavones, which are contained in nijiru supplement, stimulate the production of $\gamma$-carboxylated osteocalcin in the osteoblasts of bone tissue in healthy individuals. In addition, nijiru contains large amounts of saponin, ${ }^{14}$ ) which has been shown to stimulate bone minerazalization. ${ }^{15)}$ It is possible that saponin has a stimulatory effect on the production of $\gamma$-carboxylated osteocalcin. The intake of nijiru supplement may therefore stimulate bone mineralization in healthy individuals.

The intake of nijiru supplement caused a significant decrease in serum calcium and inorganic phosphorus concentrations in healthy volunteers. This decrease may be partly involved in bone mineralization, which is related to an increase in $\gamma$-carboxylated osteocalcin following the intake of nijiru.

Serum findings associated with liver and kidney functions were not significantly altered by the intake of nijiru supplement in healthy individuals. This indicates that prolonged intake of nijiru does not influence metabolic functions of the liver and kidney in such individuals. In addition, the body weight of the healthy volunteers did not change significantly with prolonged intake of nijiru (data not shown). From these observations, it is assumed that nijiru is a safety and healthy dietary supplement.

Nutrition may have a role in the prevention of bone loss with increasing age. Numerous elements in food have been shown to have an anabolic effect on bone metabolism..$^{21,22)}$ The isoflavone and saponin in nijiru may be related to an anabolic effect an bone mineralization in healthy individuals. The intake of nijiru as a supplement may thus have a role in the prevention of age-related bone loss.

\section{REFERENCES}

1) Cooper, C. and Melton, J., III (1992) Epidemiology of osteoporosis. Trends Endocrinol. Metab., 3, 224229.

2) Cummings, S. R., Kelsey, J. L., Nevitt, M. C. and O’Dowd, K. J. (1985) Epidemiology of osteoporosis and osteoporotic fracture. Epidemiol. Rev., 7, 178199.

3) Bonjour, J.-P., Schurch, M.-A. and Ruzzoli, R. (1996) Nutritional aspects of hip fractures. Bone, 18, 139S-144S.

4) Yamaguchi, M. and Gao, Y. H. (1997) Anabolic effect of genistein on bone metabolism in the femoral-metaphyseal tissues of elderly rats is inhibited by the anti-estrogen tamoxifen. Res. Exp. Med., 197, 101-107.

5) Yamaguchi, M. and Gao, Y. H. (1998) Anabolic effect of genistein and genistin on bone metabolism in the femoral-metaphyseal tissues of elderly rats: The genistein effect is enhanced by zinc. Mol. Cell. Biochem., 178, 377-382.

6) Gao, Y. H. and Yamaguchi, M. (1999) Anabolic effect of daidzein on cortical bone in tissue culture: Comparison with genistein effect. Mol. Cell. Biochem., 194, 93-98. 
7) Sugimoto, E. and Yamaguchi, M. (2000) Anabolic effect of genistein in osteoblastic MC3T3-E1 cells. Int. J. Mol. Med., 5, 515-520.

8) Sugimoto, E. and Yamaguchi, M. (2000) Stimulatory effect of daidzein in osteoblastic MC3T3-E1 cells. Biochem. Pharmacol., 59, 471-475.

9) Yamaguchi, M. and Sugimoto, E. (2000) Stimulatory effect of genistein and daidzein on protein synthesis in osteoblastic MC3T3-E1 cells: Activation of aminoacyl-tRNA synthetase. Mol. Cell. Biochem., 214, 97-102.

10) Yamaguchi, M. and Gao, Y. H. (1998) Inhibitory effect of genistein on bone resorption in tissue culture. Biochem. Pharmacol., 55, 71-76.

11) Gao, Y. H. and Yamaguchi, M. (1999) Inhibitory effect of genistein on osteoclast-like cell formation in mouse marrow cultures. Biochem. Pharmacol., 58, 767-772.

12) Gao, Y. H. and Yamaguchi, M. (1999) Suppressive effect of genistein on rat bone osteoclasts: Apoptosis is induced through $\mathrm{Ca}^{2+}$ signaling. Biol. Pharm. Bull., 22, 805-809.

13) Gao, Y. H. and Yamaguchi, M. (2000) Suppressive effect of genistein on rat bone osteoclasts: Involvement of protein kinase inhibition and protein tyrosine phosphatase activation. Int. J. Mol. Med., 5, 261-267.

14) Ono, R. and Yamaguchi, M. (1999) Increase in bone components of rats orally administrated isoflavonecontaining soybean extract (nijiru). J. Health Sci., 45, 66-69.

15) Ono, R. and Yamaguchi, M. (1999) Anabolic effect of soybean saponin on bone components in the femoral tissues of rats. J. Health Sci., 45, 251-255.

16) Ono, R., Ma, Z. J. and Yamaguchi, M. (2000) Prolonged intake of fermented soybean diets with supplementation of isoflavone and saponin prevents bone loss in ovariectomized rats. J. Health Sci., 46, 70-74.

17) Koyama, N., Ohara, K., Yokota, H., Kurome, J., Katayama, M., Hino, F., Kato, I. and Akai, T. (1991) A one step sandwich enzyme immunoassay for gamma-carboxylated osteocalcin using monoclonal antibodies. J. Immunol. Methods, 139, 17-23.

18) Hauschka, P. V., Lian, J. B. and Gallop, P. M. (1975) Direct identification of the calcium-binding amino acid, $\gamma$-carboxyglutamate, in mineralized tissue. Proc. Natl. Acad. Sci. U.S.A., 72, 3925-3929.

19) Price, P. A. (1985) Vitamin K-dependent formation of bone gla protein (osteocalcin) and its function. Vitam. Horm., 42, 65-108.

20) Hanschka, P. V. and Carr, S. A. (1982) Calciumdependent $\alpha$-helical structure in osteocalcin. Biochemistry, 21, 2538-2547.

21) Ma, Z. J., Shimanuki, S., Igarashi, A., Kawasaki, Y. and Yamaguchi, M. (2000) Preventive effect of dietary fermented soybean on bone loss in ovariectomized rats: Enhancement with isoflavone and zinc supplementation. J. Health Sci., 46, 263268.

22) Ma, Z. J. and Yamaguchi, M. (2000) Synergistic effect of genistein and casein phosphopeptides on bone components in young and elderly female rats. J. Health Sci., 46, 474-479. 